OPEN ACCESS

Edited by:

Peida Zhan,

Zhejiang Normal University, China

Reviewed by:

Qianqian Pan,

The University of Hong Kong,

Hong Kong

Jiwei Zhang,

Yunnan University, China

${ }^{*}$ Correspondence:

Qiao Lin

qlin7@uic.edu;

qiaolin234@gmail.com

Specialty section:

This article was submitted to

Quantitative Psychology

and Measurement,

a section of the journal

Frontiers in Psychology

Received: 21 May 2020

Accepted: 05 August 2020

Published: 11 September 2020

Citation:

Lin Q, Xing K and Park YS (2020)

Measuring Skill Growth

and Evaluating Change: Unconditional and Conditional Approaches to Latent

Growth Cognitive Diagnostic Models.

Front. Psychol. 11:2205.

doi: 10.3389/fpsyg.2020.02205

\section{Measuring Skill Growth and Evaluating Change: Unconditional and Conditional Approaches to Latent Growth Cognitive Diagnostic Models}

\author{
Qiao Lin ${ }^{1 *}$, Kuan Xing ${ }^{2}$ and Yoon Soo Park ${ }^{3}$ \\ ${ }^{1}$ Department of Educational Psychology, University of Illinois at Chicago, Chicago, IL, United States, ${ }^{2}$ University \\ of Tennessee Health Science Center, Memphis, TN, United States, ${ }^{3}$ Department of Medical Education, University of Illinois \\ at Chicago, Chicago, IL, United States
}

During the past decade, cognitive diagnostic models (CDMs) have become prevalent in providing diagnostic information for learning. Cognitive diagnostic models have generally focused on single cross-sectional time points. However, longitudinal assessments have been commonly used in education to assess students' learning progress as well as evaluating intervention effects. Thus, it becomes natural to identify longitudinal growth in skills profiles mastery, which can yield meaningful inferences on learning. This study proposes longitudinal CDMs that incorporate latent growth curve modeling and covariate extensions, with the aim to measure the growth of skills mastery and to evaluate attribute-level intervention effects over time. Using real-world data, this study demonstrates applications of unconditional and conditional latent growth CDMs. Simulation studies show stable parameter recovery and classification of latent classes for different sample sizes. These findings suggest that building on the well-established growth modeling frameworks, applications of covariate-based longitudinal CDM can help understand the effect of explanatory factors and intervention on the change of attribute mastery.

Keywords: cognitive diagnostic model, covariate extension, latent growth curve, longitudinal analysis, learning progression

\section{INTRODUCTION}

Growth of knowledge and skills are important indicators of learning, which commonly results from the implementation of interventions such as course materials, instructional curriculum, teaching methods, and policies. For educational systems and educators, it is important to understand the changes in learning by evaluating the intervention effects. To quantify these effects, longitudinal assessments or pre-and post-test designs have been widely used and the raw score has been examined to reveal the progress in learning. Longitudinal assessment designs involve repeated observations of variables over a period of time while pre-and post-test designs focus on two measurements that are taken before and after a treatment. However, simple comparison between 
time points may lack reliability and validity (Linn and Slinde, 1977); therefore, researchers may seek applying psychometric models to measure students' knowledge and aptitude that are characterized as latent constructs. Item response theory (IRT) allows psychometrically specifying students' ability as continues latent variables and has a tradition to employ longitudinal models to assess the growth in ability (Andersen, 1985; Fischer, 1989; Embretson, 1991). The multidimensional IRT models are useful to measure how the unidimensional ability increase over a period of time, yet it is hard to diagnose the increment when the latent constructs are correlated with one another over repeated measures.

In recent years, cognitive diagnostic models (CDMs), also known as diagnostic classification models (DCMs), have drawn increasing attention from researchers, as provides diagnostic information for learning and instruction (Bradshaw and Levy, 2019). Several CDMs and assessments have been developed to evaluate examinees' mastery status on a set of cognitive skills (e.g., DiBello et al., 1995; Bradshaw et al., 2014; Culpepper, 2019; Culpepper and Chen, 2019). Most commonly, CDMs have been used to assess students' skills profiles at a single time point rather than measuring changes in skills proficiency over time. However, it is important for educators to know the students' learning trajectories to achieve learning goals as well as the effects of intervention on the growth of student skills.

In this regard, latent transition analysis (LTA; Collins and Wugalter, 1992) has been incorporated to the recent development of CDM to evaluate changes in skills mastery. For example, Li et al. (2016) employed DINA model as the measurement model in an LTA to demonstrate a means of analyzing change in cognitive skills over time. Similarly, Kaya and Leite (2017) developed a model combining the LTA and deterministic input noisy "and" gate (DINA; Junker and Sijtsma, 2001) and deterministic input noisy "or" gate (DINO; Templin and Henson, 2006) CDMs to address within-individual and between-groups change in follow-up measurements of learning. In addition, Madison and Bradshaw (2018a) proposed the Transition Diagnostic Classification Model (TDCM) that combined log-linear cognitive diagnosis model (LCDM; Henson et al., 2009) and with LTA to provide a more general framework for measuring growth in cognitive diagnostic modeling. Compared to the models proposed by Li et al. (2016) and Kaya and Leite (2017) that assume specific item response structures and place constraints on parameters, TCDM use a general DCM framework that subsume early models and combine it with LTA. They further extended the TDCM to model multiple groups (MG-TDCM), thereby enabling the examination of group differential growth in attribute mastery in pre-and posttest design (Madison and Bradshaw, 2018b).

To model the learning trajectory, Wang et al. (2018) proposed a family of learning models that use higher-order, hidden Markov model (HO-HMM) to model attribute transition and incorporate CDM framework to understand individualized learning trajectory. Furthermore, Chen et al. (2018) proposed a class of dynamic CDM models to trace learning trajectories. They focused on investigating different types of learning trajectories and developed a Bayesian Modeling framework to estimate these learning trajectories. Focusing on modeling the growth in the higher-order latent trait, Lee (2017) proposed longitudinal growth curve cognitive diagnosis models (GC-CDM) that trace changes in the higher-order latent traits to incorporate learning over time into the cognitive assessment framework. Likewise, Huang (2017) embedded a multilevel structure into higher order latent traits and extended the generalized deterministic input, noisy "and" gate (G-DINA) mode to a multilevel higher order CDM, which enable the measurement of changes in the latent trait in longitudinal data. Most recently, Zhan et al. (2019) proposed a longitudinal diagnostic classification modeling approach for assessing learning growth in both repeated measures design and anchor-item design. Different from the LTA-based methods providing attribute-level transition probability matrix, the proposed longitudinal DINA model (Long-DINA) is able to provide quantitative values of overall and individual growth.

Although various longitudinal CDMs have been developed to measure the transition of examinees' attribute mastery statuses over time, fewer studies focus on the intervention effects that drive the changes in skill mastery from the perspectives of covariates extension and latent growth curve model. In this research study, we proposed two latent growth CDMs by using unconditional and conditional approaches to trace changes in latent attributes over time as well as allowing a flexible parameterization to specify covariates that can be meaningful in studying a longitudinal data structure.

Different from other longitudinal CDMs in the literature, the latent growth CDMs proposed in this study is motivated by the well-established growth curve modeling framework that are commonly used in social sciences to measure latent growth (e.g., Duncan et al., 2013). And as such, it becomes important to link the longitudinal CDM framework to existing techniques in the social sciences, to prompt more generalizable and flexible model extensions. Although some previous studies have incorporated growth curve model into CDM, they mainly focused on extending the higher-order latent trait to model the growth in learning, which assumes associations between different latent attributes, that the probability of having the skills depends on a higher order overall ability. Moreover, a focus on attributelevel changes and their impact over time can be handled and specified in the latent growth CDM framework, as proposed in this paper. In this study, we incorporate covariate extensions and latent growth curve model into the attribute-level of CDM framework instead of the higher-order latent trait level. In this way, we can monitor changes in the attribute level directly under the independence assumption for attributes probabilities.

The study consists of three parts: A real-world data analysis and two simulation studies. We first demonstrate the model application using real-world data to motivate the rationale for the latent growth framework and to monitor changes in students' skills mastery and intervention effects. The ensuing sections include two simulation studies conducted separately to examine the parameter recovery of the proposed models. In this manner, the simulation studies with varying longitudinal design components provide comprehensive inference for different number of time points, sample sizes, and covariate specification conditions. Findings from this study could help researchers 
apply the latent growth CDMs in practice and promote the development of longitudinal CDMs.

\section{COGNITIVE DIAGNOSTIC MODELS}

Cognitive diagnostic models were designed to classify examinees into skill profiles that indicate their mastery in fine-grained skills or attributes based on their performance on a set of items (Rupp et al., 2010). It refers to a class of psychometric models where patterns of attributes mastery have been represented as latent classes. Distinguish from IRT models that latent traits are continuous, CDMs examine categorical latent traits. Different kinds of CDMs have been developed in literature and various generalizations of CDMs have also been proposed including the LCDM (Henson et al., 2009), general diagnostic model (GDM; von Davier, 2008), and the generalized DINA model (G-DINA; de la Torre, 2011).

\section{Reparameterized DINA (RDINA) Model}

In this study, we use the DINA model to demonstrate the framework, which can be applied to other CDM families and generalizations of DINA models (e.g., von Davier, 2008; de la Torre, 2011). The DINA model is developed with the idea that in order to answer an item $j$ correctly, the examinee $i$ must have mastered all of the required skills (Tatsuoka, 1985). The binary latent variable $\eta_{i j}=\prod_{k=1}^{k} \alpha_{i k}^{q_{i k}}$ indicate whether the $i$ th examinee has mastered the set of attributes $\underline{\alpha}$ [i.e., attributes, $\left.\underline{\alpha}=\left(\alpha_{1}, \alpha_{2}, \ldots, \alpha_{k}\right)^{\prime}\right]$ to solve the $j$ th item, where $\eta_{i j}=1$ means the presence of the necessary set of attributes, and $\eta_{i j}=0$ otherwise. As specified by the Q-matrix, $q_{i k}$ is either zero or one, indicating whether the attribute $k$ is required for solving item $j$. This study uses the reparameterized deterministic inputs noisy "and" gate (DeCarlo, 2011) to apply the longitudinal framework, as it facilitates incorporating covariates as intervention effects in latent growth curve model. The RDINA takes the logit of the traditional DINA model

$$
\operatorname{logit} p\left(Y_{i j}=1 \mid \eta_{i j}\right)=f_{j}+d_{j} \eta_{i j}
$$

The $f_{j}$ parameter indicates the log odds of a false alarm that is the probability of getting item $j$ correct given the examinee do not have the requisite skills. The parameter $d j$ provides a measure of how well the item can discriminate an examinee with or without the mastery of required skills. The guessing and slip parameters used in the DINA model can be recovered by exponentiating the RDINA parameters (DeCarlo, 2011)as:

$$
\begin{gathered}
g_{j}=\exp \left(f_{j}\right) /\left[1+\exp \left(f_{j}\right)\right] \\
s_{j}=1-\exp \left(f_{j}+d_{j}\right) /\left[1+\exp \left(f_{j}+d_{j}\right)\right]
\end{gathered}
$$

\section{Covariate Extension to the RDINA Model}

Various latent class models have incorporated covariates as extensions (Dayton and Macready, 1988; DeCarlo, unpublished). In the DINA model, covariates can be specified either at the item level and/or attribute level. Park and Lee (2014) proposed a covariate extension to the RDINA model by applying a latent class regression framework. In particular, when a discrete or a continuous covariate, $\underline{Z}$, is introduced into a latent class model, an examinee's response probability can be expressed as

$$
p\left(Y_{i 1}, Y_{i 2}, \ldots, Y_{i j} \mid \underline{Z}\right)=\sum_{\underline{\alpha}} p(\underline{\alpha} \mid \underline{Z}) \prod_{j} p\left(Y_{i j} \mid \underline{\alpha}, \underline{Z}\right)
$$

where $p\left(Y_{i 1}, Y_{i 2}, \ldots, Y_{i j} \mid \underline{Z}\right)$ represents response probability conditioning on covariate $\underline{Z}, p(\underline{\alpha} \mid \underline{Z})$ represents the covariate affecting the attribute probability, $p\left(Y_{i j} \mid \underline{\alpha}, \underline{Z}\right)$ represents the covariate affecting the response probability. In particular, the effects of the covariate on the response probability and attribute probability are shown as following:

$$
\begin{gathered}
\operatorname{logit} p\left(Y_{i j} \mid \underline{\alpha}, \underline{Z}\right)=f_{j}+d_{j} \eta_{i j}+l_{j} \underline{Z} \\
\operatorname{logit} p\left(\alpha_{k} \mid \underline{Z}\right)=b_{k}+h_{k} \underline{Z}
\end{gathered}
$$

where the parameter $l_{j}$ reflects the changes in the guessing and slip parameter for a unit change in $\underline{Z}$. Similarly, the parameter $h_{k}$ indicates the changes in the attribute difficulty parameter $\left(b_{k}\right)$, when the covariate $\underline{Z}$ is conditional on the attribute level.

\section{Relationship Between RDINA and General Diagnostic Model}

The RDINA model was employed in this study to establish the framework; however, the parameterization used in its covariate extension can be extended and reparameterized as special cases of the GDM (von Davier, 2005; Park and Lee, 2019). In the GDM, the observed response $X$ is modeled for $i$ items, $\mathrm{x}$ response categories, and $j$ respondents as follows:

$$
P(X=x \mid i, j)=\exp \left[f\left(\lambda_{x i}, \theta_{j}\right)\right] /\left\{1+\sum_{m} \exp \left[f\left(\lambda_{x i}, \theta_{j}\right)\right]\right\}
$$

GDM item parameters are the $\lambda_{x i}=\left(\beta_{x i}, \boldsymbol{q}_{\mathrm{i}}, \gamma_{x i}\right)$, which include slope parameters and the Q-matrix specification, $q_{\mathbf{i}}$. In the DINA where attributes are binary, the skill vector for examinee $j, \theta_{j}=$ $\left(\alpha_{j 1}, \ldots, \alpha_{j k}\right)$, are binary values. As shown in von Davier (2014, p. 58), the DINA can be parameterized as a special case of the GDM as follows:

$$
P\left(X_{v i}=1 \mid \boldsymbol{q}_{i,}^{*}, \boldsymbol{a}^{*}\right)=\frac{\exp \left(\beta_{i}+\sum_{k} \gamma_{i k} a_{k}^{*} q_{i k}^{*}\right)}{1+\exp \left(\beta_{i}+\sum_{k} \gamma_{i k} a_{k}^{*} q_{i k}^{*}\right)}
$$

When a covariate $\mathrm{Z}$ introduced to Eq. 5, the following $h_{k}$ parameters are added:

$$
P\left(X_{v i}=1 \mid \boldsymbol{q}_{i,}^{*}, \boldsymbol{a}^{*}, Z\right)=\frac{\exp \left(\beta_{i}+\sum_{k} \gamma_{i k} a_{k}^{*} q_{i k}^{*}+h_{k} Z\right)}{1+\exp \left(\beta_{i}+\sum_{k} \gamma_{i k} a_{k}^{*} q_{i k}^{*}+h_{k} Z\right)}
$$

Taking the logit simplifies the model to the item-level of covariate extension approach as presented in Eq. 4.

\section{Latent Growth Curve Model}

Latent growth curve model has been widely used in longitudinal analysis to estimate growth over time, such as examining 
the treatment effects in the pre-post intervention study. As a special case of structural equation model (SEM), latent growth modeling formwork extend SEM to represent repeated measures of dependent variables as a function of time and other measures. Based on the research of Tucker (1958), Rao (1958), Meredith and Tisak (1984) and Meredith and Tisak (1990) furthered SEM to model the interindividual differences in change. To model the changes in a variable over time, latent growth curve model assumes that there is a systematic trajectory of change underlying the repeated measures of the variable. In particular, for $i(i=1,2, \ldots, n)$ subjects measured at $j(j=1,2, \ldots, t)$ occasions, the measurement model of latent growth curve model can be expressed as

$$
y_{i j}=\lambda_{0 j} \eta_{0 i}+\lambda_{1 j} \eta_{1 i}+\varepsilon_{i j}
$$

where $y_{i j}$ is the outcome variable for individual $i$ at time $j . \eta_{0 i}$ and $\eta_{1 i}$ represent latent trajectory parameters: individual's initial level (i.e., intercept) and rate of change over time (i.e. slopes). $\varepsilon_{i j}$ represent time-specific error for person $i$. In the structural model of latent growth curve model, these latent trajectory parameters become outcome variables that can be expressed as:

$$
\begin{aligned}
& \eta_{0 i}=\mu_{0}+e_{0 i} \\
& \eta_{1 i}=\mu_{1}+e_{1 j}
\end{aligned}
$$

where $\mu_{0}$ represents the sample mean initial level, $e_{0 i}$ represents the deviations from mean initial level for individual $i ; \mu_{1}$ represents the sample mean rate of change, $e_{1 i}$ represents the deviations from mean rate of change for individual $i$.

In latent growth curve model, $\lambda_{0 j}$ is fixed at 1 for all $j$ occasions. It should be noted that the equations presented above are considered as an unconditional latent growth curve model because there is no covariate involved. A conditional latent growth curve model that contains covariates can be specified by adding predictors of the outcome variable into Eq 8 . The corresponding covariates effects on the latent trajectory parameters could be included in Eqs 8.1 and 8.2.

\section{The Latent Growth Cognitive Diagnostic Model}

Motivated from latent growth curve models and RDINA model with covariate extensions, we propose two latent growth curve CDMs (LG-CDMs) using unconditional and conditional approaches to track the changes in examinees' latent attributes as well as evaluating the effects of covariate at the latent attributes level. For the LG-CDMs, an examinee is response probability $p\left(Y_{i j}=1 \mid \eta_{i j}\right)$ was specified by the RDINA model, which was shown in the above Eq. 1.

\section{Unconditional LG-CDM}

Motivated from the unconditional latent growth curve model with random intercept and random slope, we develop an Unconditional LG-CDM that includes unconditional latent growth curve to the attribute level of CDM framework as linear model. At the attribute level $\underline{\alpha}_{k}=\left(\alpha_{1}, \alpha_{2}, \ldots, \alpha_{k}\right)^{\prime}$, we assume a linear relationship between time and attributes to model the changes in attributes over time. When no covariate is specified,
Eq. (9) shows the latent growth model with time effect on the probabilities of the $K$ attributes $p\left(\alpha_{k} \mid\right.$ time $)$ as

$$
\operatorname{logit} p\left(\alpha_{k} \mid \text { time }\right)=b_{k}+\gamma_{k}(\text { time })+\zeta_{k}+\varepsilon_{t k}, \quad \varepsilon_{t k} \sim N(0,1)
$$

Equation 9 represents time is conditioned on the attributes probability, which can be viewed as a predictor of the attribute patterns. Following the interpretation of the RDINA model with covariate extension, $b_{k}$ represents the fixed-effect attributes difficulty parameter. $\zeta_{k}$ represents the random intercept parameter for attribute $k$, which allows estimation for each attribute, accounting for individual examinee differences at baseline. Similarly, $\gamma_{k}$ represents the random slope parameter for attribute $k$, which allows differences in examinee rates of growth. $\varepsilon_{t k}$ represents time-specific error for attribute $k$. Eq. 9.1 shows the associated equation for the random intercept that follows a normal distribution with mean $\mu_{0 k}$ and variance of $\sigma_{0 k}$. Eq. 9.2 shows the association equation for the random slope that also follows a normal distribution with mean $\mu_{1 k i}$ and variance of $\sigma_{1 k} . e_{0 k}$ and $e_{1 k}$ represent the deviations from mean initial level and mean rate of change for attribute $k$, which are random-effect parameters introduced by the latent growth curve model. In this study, the mean and variance of both random effects are fixed to 0 and 1 , respectively.

$$
\begin{aligned}
& \zeta_{k}=\eta_{0 k}=\mu_{0 k}+e_{0 k} \\
& \gamma_{k}=\eta_{1 k}=\mu_{1 k}+e_{1 k}
\end{aligned}
$$

Where $\eta_{0}$ and $\eta_{1}$ represent latent trajectory parameters: individual's initial level and rate of change over time, which was specified in the latent growth curve model framework.

\section{Conditional LG-CDM}

In addition, we also propose a Conditional LG-CDM based on the conditional latent growth curve model with random intercept and random slope to evaluate the effects of covariate (e.g., intervention effect) on changes in the attribute level. In particular, covariate vector $\underline{Z}$, is introduced into the latent growth curve model effecting on the random effects. $\underline{Z}$ could be either discrete or continuous covariate. Equation (10) represents the latent growth model with both time and covariate effects on the attribute probability $p\left(\alpha_{k} \mid\right.$ time, $\left.\underline{Z}\right)$ as

$$
\begin{aligned}
\operatorname{logit} p\left(\alpha_{k} \mid \text { time }, \underline{Z}\right)= & b_{k}+\gamma_{k}(\text { time })+h_{k}(\underline{Z})+\zeta_{k}+\varepsilon_{t k}, \\
& \varepsilon_{t k} \sim N(0,1)
\end{aligned}
$$

The interpretation of Conditional LG-CDM is similar to the Unconditional LG-CDM that $b_{k}$ represents the attributes difficulty parameter, $\zeta_{k}$ represents the random intercept parameter, and $\gamma_{k}$ represents the random slope parameter. Both random effects follow the normal distribution with fixed mean of 0 and variance of 1 for model identification. $\varepsilon_{t k}$ represents time-specific error for attribute $k$. What's more, the parameter $h_{k}$ represents the regression coefficient of the covariate vector $\underline{Z}$, which reflects the shift in the attribute difficulty $b_{k}$, random intercept $\zeta_{k}$ and random slope $\gamma_{k}$ when the covariate is present to affect the attribute. Specifically, Eq. 10.1 shows the term for the 
respective covariate coefficient $\left(h_{0 k}\right)$ affecting random intercept parameter for each attribute $\mathrm{k}$. Equation 10.2 shows the term for the respective covariate coefficient $\left(h_{1 k}\right)$ affecting random slope parameter for each attribute $\mathrm{k}$.

$$
\begin{aligned}
& \zeta_{k}=\eta_{0 k}+h_{0 k}(\underline{Z}) \\
& \gamma_{k}=\eta_{1 k}+h_{1 k}(\underline{Z})
\end{aligned}
$$

Likewise, $\eta_{0 k}$ and $\eta_{1 k}$ represent attribute's initial level and rate of change, which can be extended as shown in Eqs 8.1 and 8.2.

From the perspective of multilevel model, Unconditional LGCDM and Conditional LG-CDM can be viewed as three-level model where the first level is the time level that involves multiple repeated measures of the same examinee (shown in Eqs 9.1 and 9.2; Eqs 10.1 and 10.2). The second level is the item at individual level that the RDINA model was used to specify an examinee i's item response (shown in Eq. 1). And the third level is the attribute at individual level that the latent growth curve model was used to specify the change of attributes over time and the covariate effect (Eqs 9 and 10).

In addition, time effect could be specified at the item level as well. Modeled with the RDINA, we let $Y_{i j t}$ be an examinee $i$ 's response for item $j$ at time $t$, given the binary latent variable $\eta_{i j t}$ and time. The response probability of a person $i$ getting item $j$ correctly at time $t$ is shown as following:

$$
\begin{aligned}
\operatorname{logit} p\left(Y_{i j t}=1 \mid \eta_{i j t,} \text { time }\right) & =f_{j}+d_{j} \eta_{i j t}+\rho_{j}(\text { time })+\xi_{i j t}+\varphi_{i j t} \\
\xi_{i j t} & =\eta_{0 i}=\mu_{0}+e_{0 i} \\
\rho_{j} & =\eta_{1 i}=\mu_{1}+e_{1 i}
\end{aligned}
$$

where $\xi_{i j t}$ represents the random intercept parameter that allows variation in baseline for item response probability $p\left(Y_{i j t}=1 \mid \eta_{i j t}\right.$, time). The $\rho_{j}$ represents the random slope parameter that allows growth rate of item response probability vary across time. $\varphi_{i j t}$ is the error term.

Taken together, this study focuses on applying the latent growth curve model into the attribute-level of CDM to analyze how the attributes mastery change over time. There are several advantages to using the Unconditional LG-CDM and Conditional LG-CDM, including that they directly estimate the learning trajectory parameters on the attribute level but also allow covariates effects involved to estimate the intervention effects simultaneously.

\section{REAL-WORLD DATA ANALYSIS}

\section{Methods}

Real-world data analysis was conducted to motivate the potential of the LG-CDMs and demonstrate its application. We used the pretest and posttest data of a mathematics test $(N=879)$ in the real-world analysis. The mathematic test was developed in a large scale education study that investigated the difficulties for the disabled students in solving mathematic problems (Bottge et al., 2014, 2015). Specifically, an instructional method of
Enhanced Anchored Instruction (EAI) was employed in the study to help improve the mathematics achievement of disabled students (Bottge et al., 2003). The design of cluster-randomized controlled trial was used to assign clusters of middle school students to a treatment group or a control group. In the treatment group, students received EAI video sessions about mathematics problem-solving and in the control group, students received instruction method as usual. To evaluate effectiveness of EAI instructional method, the mathematics test was administrated before and after the instructional period and the assessment data was collected. Thus, the dataset consisted of 21-item responses to the test measured four attributes over two time points. A Q-matrix was identified for the four attributes that corresponded to the four instructional units, including $\alpha_{1}$ : ratios and proportional relationships, $\alpha_{2}$ : measurement and data, $\alpha_{3}$ : number systems (fractions), and $\alpha_{4}$ : geometry (graphing). Each test item was mapped to one attribute (Appendix). Two latent growth curve CDMs were fit, Unconditional LG-CDM and Conditional LG-CDM.

Data analyses were conducted using Latent GOLD 5.0 (Vermunt and Magidson, 2013).

Two statistics were used to examine attribute classification (1) proportion correctly classified $\left(P_{c}\right)$ and (2) Lambda $(\lambda)$ (Clogg, 1995), where both are based on the maximum posterior probability to examine the quality of classification. In particular, Eq. (12) shows the use of estimated posterior probabilities in obtaining an estimate of the expected proportion of cases correctly classified for attribute $k\left(\alpha_{k}\right)$ :

$$
P_{c}=\sum_{s}\left[n_{s} \times \max p\left(\alpha_{k} \mid \underline{Y_{i 1}}, \underline{Y_{i 2}}, \ldots, \underline{Y_{i J}}\right)\right] / N
$$

where $s$ represents each unique response pattern, $n_{s}$ is the frequency of each pattern (i.e., number of cases with a particular pattern), $\max p\left(\alpha_{k} \mid \underline{Y_{i 1}}, \underline{Y_{i 2}}, \ldots, \underline{Y_{i J}}\right)$ represents the maximum posterior probability for a given response pattern vector $\left(\underline{Y_{i 1}}, Y_{i 2}, \ldots, \underline{Y_{i J}}\right), N$ is the total number of cases in a latent class response pattern.

$\lambda$ makes a correction for classification that can occur by chance, which can be expressed as

$$
\lambda=\frac{P_{c}-\max p\left(\alpha_{k}\right)}{1-\max p\left(\alpha_{k}\right)}
$$

where $p\left(\alpha_{k}\right)$ represents the latent class size with $p\left(\alpha_{k}\right)>0$. Meanwhile, $\lambda$ also reflects the relative reduction in classification error (Kruskal and Goodman, 1954; Clogg, 1995).

Both expectation-maximization (EM) and Newton-Raphson algorithms were used to obtain maximum likelihood (ML) or posterior mode (PM) estimates. The PM estimation uses a prior distribution to smooth solutions that are near the boundary of the parameter space. Therefore, this method can avoid a boundary estimation issues that are commonly associated with latent class models (DeCarlo, 2011). In addition, to avoid problems of local maxima, 100 sets of starting values were used to obtain the global maximum. Finally, to check for local identification, the rank of the Jacobian matrix was examined to be of full rank as specified 
as a required condition for local identification in latent class regression models (Huang and Bandeen-Roche, 2004).

\section{Results \\ Model Fit and Classification}

Real-world data converged successfully for the two models, unconditional latent growth curve CDM (Unconditional LG$\mathrm{CDM}$ ) and conditional latent growth curve CDM (Conditional LG-CDM). Table 1 shows the classification results. The results show that the $P_{c}$ estimates are the same for the two LG-CDMs across four mathematics attributes, indicating a satisfactory classification as all statistics are greater than 0.91 . For example, with $P_{c}$ of 0.96 for attribute 2 , one would expect that $96 \%$ of the cases would be correctly classified into attribute 2 . However, it should be noted that if simply classifies all the cases into the attribute with the largest size, then the correctly classification can be achieved but may due to the chance. $\lambda$ provides a correction for this situation when calculating the proportion correctly classified. The results show that $\lambda$ are similar for the four attributes across two models and are slightly lower than $\mathrm{P}_{c}$. For example, the $\lambda$ classification on attribute 1 in Conditional LG$\mathrm{CDM}$ is 0.70 , indicating that the proportion correctly classified increase $70 \%$ by using the observers' response pattern over simply classifying all cases into the attribute with the largest size.

\section{Attribute Prevalence}

Table 2 shows the attribute prevalence for the four attributes. The attribute prevalence represents the latent class sizes of the four attributes (DeCarlo, 2011). Overall, the attribute prevalence was consistent across the two models. The probabilities of all attributes prevalence are above 0.50 , indicating that more than half of the students mastered each of the attribute. The attribute prevalence for Attribute 2 (Measurement and Data)

TABLE 1 | Classification: proportion correctly classified $\left(P_{C}\right)$ and Lambda $(\lambda)$.

\begin{tabular}{|c|c|c|c|c|c|}
\hline \multirow[t]{2}{*}{ Models } & \multirow[t]{2}{*}{ Classification } & \multicolumn{4}{|c|}{ Attributes } \\
\hline & & $\alpha_{1}$ & $\alpha_{2}$ & $\alpha_{3}$ & $\alpha_{4}$ \\
\hline \multirow[t]{2}{*}{ Unconditional LG-CDM } & $P_{c}$ & 0.91 & 0.96 & 0.92 & 0.93 \\
\hline & $\lambda$ & 0.79 & 0.90 & 0.84 & 0.82 \\
\hline \multirow[t]{2}{*}{ Conditional LG-CDM } & $P_{c}$ & 0.91 & 0.96 & 0.92 & 0.93 \\
\hline & $\lambda$ & 0.70 & 0.90 & 0.84 & 0.82 \\
\hline
\end{tabular}

TABLE 2 | Attribute prevalence.

\begin{tabular}{lcccc}
\hline Model & $\alpha_{1}$ & $\alpha_{2}$ & $\alpha_{3}$ & $\alpha_{\mathbf{4}}$ \\
\hline Unconditional LG-CDM & $0.57(0.03)$ & $0.62(0.02)$ & $0.53(0.03)$ & $0.62(0.08)$ \\
Conditional LG-CDM & $0.57(0.03)$ & $0.62(0.02)$ & $0.53(0.03)$ & $0.62(0.02)$
\end{tabular}

Values in parenthesis are standard errors. Attributes based on the EAl instruction are as follows: $\alpha_{1}$ : Ratios and Proportional Relationships; $\alpha_{2}$ : Measurement and Data; $\alpha_{3}:$ Number Systems (Fractions); $\alpha_{4}$ : Geometry (Graphing). and Attribute 4 (Geometry) had slightly higher probabilities than the attribute prevalence for Attribute 1 (Ratios and Proportional Relationships) and Attribute 3 (Number Systems).

\section{Item Parameters}

Table 3 shows the item parameters $\left(f_{j}\right.$ and $\left.d_{j}\right)$ for the Unconditional LG-CDM and Conditional LG-CDM, which are estimated from the RDINA model. Calculating the exponential of the $f_{j}$ and $d_{j}$ parameters, one can obtain guessing and slip parameters of the DINA model (shown in Eqs 1.1 and 1.2). Overall, the item parameters are consistent across the two models. Derived from Eqs 1.1 and 1.2, the average guessing and slip parameters estimates for Unconditional LG-CDM were 0.22 and 0.35; for Conditional LG-CDM were 0.22 and 0.36 . With respect to each attribute, the average guessing and slip parameters were respectively the same across the two models: for the attribute of Ratios and Proportional Relationships were 0.14 and 0.49; for the attribute of Measurement and Data were 0.28 and 0.23 ; for the attribute of Number Systems (Fractions) were 0.28 and 0.23 ; for the attribute of Geometry (Graphing) were 0.25 and 0.36 . In particular, for both Unconditional LG-CDM and Conditional LG-CDM, Item 2 (Attribute of Measurement and Data) has the greatest guessing estimates while Item 17 (Attribute of Ratios and Proportional Relationships) has the greatest slip estimates.

\section{Attribute Parameters and Growth}

Table 4 summarized the attribute-level parameters for the Unconditional LG-CDM and Conditional LG-CDM, which

TABLE 3 | Item parameters for Unconditional and Conditional LG-CDM.

\begin{tabular}{|c|c|c|c|c|c|}
\hline \multirow[b]{2}{*}{ Item } & \multirow[b]{2}{*}{ Attributes } & \multicolumn{2}{|c|}{ Unconditional LG-CDM } & \multicolumn{2}{|c|}{ Conditional LG-CDM } \\
\hline & & $f_{j}$ & $d_{j}$ & $f_{j}$ & $d_{j}$ \\
\hline$Y_{1}$ & $\alpha_{1}$ & $-2.09(0.02)$ & $1.97(0.17)$ & $-2.10(0.16)$ & $1.98(0.17)$ \\
\hline$Y_{2}$ & $\alpha_{2}$ & $-0.24(0.08)$ & $2.06(0.13)$ & $-0.24(0.08)$ & $2.06(0.13)$ \\
\hline$Y_{3}$ & $\alpha_{2}$ & $-2.61(0.17)$ & $1.92(0.19)$ & $-2.62(0.18)$ & $1.92(0.19)$ \\
\hline$Y_{4}$ & $\alpha_{3}$ & $-1.87(0.13)$ & $1.87(0.14)$ & $-1.88(0.13)$ & $1.87(0.14)$ \\
\hline$Y_{5}$ & $\alpha_{3}$ & $-1.14(0.11)$ & $2.34(0.13)$ & $-1.14(0.11)$ & $2.34(0.13)$ \\
\hline$Y_{6}$ & $\alpha_{3}$ & $-0.74(0.09)$ & $1.88(0.12)$ & $-0.74(0.09)$ & $1.89(0.12)$ \\
\hline$Y_{7}$ & $\alpha_{3}$ & $-3.73(0.42)$ & $3.57(0.41)$ & $-3.75(0.43)$ & $3.58(0.42)$ \\
\hline$Y_{8}$ & $\alpha_{3}$ & $-0.96(0.09)$ & $1.14(0.12)$ & $-0.96(0.09)$ & $1.14(0.12)$ \\
\hline$Y_{9}$ & $\alpha_{2}$ & $-0.76(0.09)$ & $2.21(0.12)$ & $-0.76(0.09)$ & $2.21(0.12)$ \\
\hline$Y_{10}$ & $\alpha_{2}$ & $-0.97(0.10)$ & $2.84(0.13)$ & $-0.97(0.10)$ & $2.84(0.13)$ \\
\hline$Y_{11}$ & $\alpha_{2}$ & $-0.59(0.09)$ & $3.09(0.15)$ & $-0.59(0.09)$ & $3.09(0.15)$ \\
\hline$Y_{12}$ & $\alpha_{2}$ & $-1.38(0.11)$ & $3.03(0.14)$ & $-1.38(0.11)$ & $3.02(0.14)$ \\
\hline$Y_{13}$ & $\alpha_{1}$ & $-0.64(0.10)$ & $2.01(0.13)$ & $-0.63(0.10)$ & $1.99(0.13)$ \\
\hline$Y_{14}$ & $\alpha_{1}$ & $-2.61(0.23)$ & $2.93(0.22)$ & $-2.60(0.22)$ & $2.91(0.22)$ \\
\hline$Y_{15}$ & $\alpha_{4}$ & $-0.99(0.10)$ & $1.91(0.13)$ & $-0.99(0.10)$ & $1.91(0.13)$ \\
\hline$Y_{16}$ & $\alpha_{4}$ & $-0.70(0.10)$ & $1.82(0.12)$ & $-0.70(0.10)$ & $1.82(0.12)$ \\
\hline$Y_{17}$ & $\alpha_{1}$ & $-3.41(0.27)$ & $1.94(0.29)$ & $-3.49(0.28)$ & $2.02(0.30)$ \\
\hline$Y_{18}$ & $\alpha_{4}$ & $-0.27(0.09)$ & $2.35(0.14)$ & $-0.27(0.09)$ & $2.35(0.14)$ \\
\hline$Y_{19}$ & $\alpha_{4}$ & $-1.77(0.13)$ & $1.96(0.15)$ & $-1.76(0.13)$ & $1.96(0.15)$ \\
\hline$Y_{20}$ & $\alpha_{4}$ & $-1.41(0.11)$ & $1.47(0.13)$ & $-1.41(0.11)$ & $1.47(0.13)$ \\
\hline$Y_{21}$ & $\alpha_{4}$ & $-1.92(0.14)$ & $1.63(0.15)$ & $-1.93(0.14)$ & $1.64(0.15)$ \\
\hline
\end{tabular}

Values in parenthesis are standard errors. Attributes based on the EAl instruction are as follows: $\alpha_{1}$ : Ratios and Proportional Relationships; $\alpha_{2}$ : Measurement and Data; $\alpha_{3}:$ Number Systems (Fractions); $\alpha_{4}$ : Geometry (Graphing). 
include attribute difficulty $\left(b_{k}\right)$, intercept and slope of latent growth curve $\left(\eta_{0}\right.$ and $\left.\eta_{1}\right)$, and regression coefficients $\left(h_{k}\right)$ for the intervention effect. In general, the estimates of random intercept and random slope were very close, indicating that the examinees' initial level and growth rate are similar for the two models. As the Conditional LG-CDM incorporates covariate in addition to the Unconditional LGCDM, regression coefficients $\left(h_{k}\right)$ of the covariate indicate shifts in the attributes difficulty as a result of the treatment effect (EAI). Results show that the parameter estimates of four attributes difficulty are all lower in the Conditional LG-CDM than the Unconditional LG-CDM, suggesting that all attributes were easier to be mastered when involving the treatment effect. In particular, Attribute 1 (Ratios and Proportional Relationships) and Attribute 4 (Geometry) yielded greater differences between the two models then Attribute 2 (Measurement and Data) and Attribute 3 (Number Systems), with a difference of 0.34 and 0.37 units. While most treatment effects were not significant, Attribute 4 (Geometry) had significant treatment effect, shifting the difficulty parameter by 0.24 units.

\section{SIMULATION STUDY I}

\section{Methods}

Simulation studies were conducted to evaluate parameter recovery and classification of the two models: (a) Unconditional LG-CDM; (b) Conditional LG-CDM. In simulation study 1,

TABLE 4 | Attribute difficulty, growth curve and intervention effect parameters.

\begin{tabular}{|c|c|c|c|c|}
\hline \multirow[b]{2}{*}{ Parameter } & \multicolumn{2}{|c|}{ Unconditional LG-CDM } & \multicolumn{2}{|c|}{ Conditional LG-CDM } \\
\hline & Estimate & $p$ value & Estimate & $p$ value \\
\hline$\eta_{0}$ (Random Intercept) & $0.44(0.11)$ & & $0.46(0.15)$ & \\
\hline$\eta_{1}$ (Random Slope) & $0.04(0.00)$ & & $0.03(0.00)$ & \\
\hline $\begin{array}{l}h_{\text {Ok }} \text { (Treatment Effect on } \\
\text { random intercept) }\end{array}$ & - & - & $0.30(0.19)$ & 0.13 \\
\hline $\begin{array}{l}h_{1 \mathrm{k}} \text { (Treatment Effect on } \\
\text { random slope) }\end{array}$ & - & - & $0.09(0.00)$ & $<0.001$ \\
\hline$b_{1}$ (Attribute 1 Difficulty) & $-0.11(0.09)$ & & $-0.45(0.11)$ & \\
\hline $\begin{array}{l}h_{1} \text { (Treatment Effect for } \\
\text { Attribute 1) }\end{array}$ & - & - & $0.40(0.27)$ & 0.13 \\
\hline$b_{2}$ (Attribute 2 Difficulty) & $0.16(0.07)$ & & $-0.04(0.09)$ & \\
\hline $\begin{array}{l}h_{2} \text { (Treatment Effect for } \\
\text { Attribute 2) }\end{array}$ & - & - & 0.09 (.25) & 0.72 \\
\hline$b_{3}$ (Attribute 3 Difficulty) & $-0.23(0.08)$ & & $-0.44(0.10)$ & \\
\hline $\begin{array}{l}h_{3} \text { (Treatment Effect for } \\
\text { Attribute } 3 \text { ) }\end{array}$ & - & - & $0.02(0.26)$ & 0.95 \\
\hline$b_{4}$ (Attribute 4 Difficulty) & $0.16(0.04)$ & & $-0.11(0.04)$ & \\
\hline $\begin{array}{l}h_{4} \text { (Treatment Effect for } \\
\text { Attribute } 4)\end{array}$ & - & - & $0.24(0.00)$ & $<0.001$ \\
\hline
\end{tabular}

(1) Parameter $h_{k}$ indicates shift in the attributes difficulty due to mastery in treatment effect (EAl), based on the Conditional LG-CDM model. (2) Values in parenthesis represent standard errors. (3) Attributes based on the EAl instruction are as follows: $\alpha_{1}$ : Ratios and Proportional Relationships; $\alpha_{2}$ : Measurement and Data; $\alpha_{3}$ : Number Systems (Fractions); $\alpha_{4}$ : Geometry (Graphing). the EAI real-world results were used as generating population (true) values. Following the data structure of the real-world data example, the 21-item response data were generated for two time points; four attributes and Q-matrix were specified as well. Two sample size of 1000 and 2000 were examined across a specification of the four attributes. Therefore, the simulation study includes a total of four simulation conditions $(=2$ models $\times 2$ sample size conditions).

Data were generated and fit using Latent GOLD 5.0 (Vermunt and Magidson, 2013). One hundred replications were fitted for each condition. Parameters were estimated using PM estimation and the parameter recovery were evaluated for each condition using three measures: (a) Bias, (b) \% Bias, and (c) mean square error (MSE). Here, Bias $(x)=\frac{1}{N} \sum_{n=1}^{N}\left[\widehat{e_{n}}(x)-e(x)\right]$, \% Bias $=$ $|\operatorname{Bias}(x) / e(x)| \times 100 \%, \operatorname{MSE}(x)=\frac{1}{N} \sum_{n=1}^{N}\left[\widehat{e_{n}}(x)-e(x)\right]^{2}$, where $x$ is an arbitrary indicator of a parameter, $\mathrm{e}(\mathrm{x})$ is the generating (true) parameter value, and $\widehat{e_{n}}(x)$ is the $n$th replicate estimate of parameter $x$ among a total of $N=100$ replications. Similar to the real-world data analysis, we set up 100 starting values, and the Jacobian matrix was examined to be of full rank for local identification. EM (expectation-maximization) and NewtonRaphson algorithms were used to avoid a boundary estimation issue using PM estimation. The syntax of unconditional and conditional models is available from the authors upon request.

\section{Results}

\section{Parameter Recovery}

Table 5 shows the parameter recovery results (Bias, \% Bias, and MSE) by sample size $(1,000$ and 2,000$)$ for the two models. Overall, the parameter recovery revealed consistent estimates. Percent bias for item-level parameters were very close in the two models except item discrimination parameter $d j$ was slightly higher in the Conditional LG-CDM model. The parameter $d j$ provides a measure of how well the item can discriminate an examinee with or without the mastery of required skills. The overall \% bias associated with random effects were all less than $2.0 \%$ regardless of models. In Conditional LG-CDM, \% bias of random effects were lower $(\leq 1.0 \%)$. The intervention effect on attributes in the conditional model had \% bias of $37.4 \%$ when sample size is 1000 . However, it dramatically dropped to $4 \%$ when the sample size increase to 2000 . The $\%$ bias of attribute difficulty parameter are lower in the Unconditional LG-CDM model (6.7 and $3 \%$ at the sample sizes of 1,000 and 2,000).

\section{Classification}

Simulation classification indices were summarized in Table 6. The results of the two LG-CDM models agree very much. Both $P_{c}$ and $\lambda$ showed excellent classification rates on the four attributes in the two models. The classification index was little influenced by the sample sizes.

\section{Cross Fitting of Simulated Data to Other Models}

What's more, a cross fitting analysis was conducted to examine the consequences on parameter estimates and classification on fitting incorrect models. Data were generated using the 
TABLE 5 | Simulation I results: parameter recovery.

\begin{tabular}{|c|c|c|c|c|c|c|c|c|}
\hline \multirow[t]{2}{*}{ Model } & \multirow[t]{2}{*}{ Level } & \multirow[t]{2}{*}{ Parameter } & \multicolumn{3}{|c|}{ Sample $n=1,000$} & \multicolumn{3}{|c|}{ Sample $n=2,000$} \\
\hline & & & Bias & $\%$ Bias & MSE & Bias & $\%$ Bias & MSE \\
\hline \multirow[t]{4}{*}{ Unconditional LG-CDM } & Random Effects & $\lambda$ & 0.007 & $1.8 \%$ & 0.002 & -0.002 & $0.5 \%$ & 0.001 \\
\hline & Attribute Difficulty & $b_{k}$ & -0.002 & $6.7 \%$ & 0.002 & 0.000 & $3.1 \%$ & 0.001 \\
\hline & Item & $f_{j}$ & -0.013 & $1.6 \%$ & 0.024 & -0.001 & $0.6 \%$ & 0.007 \\
\hline & & $d_{j}$ & 0.015 & $0.9 \%$ & 0.030 & 0.002 & $0.4 \%$ & 0.009 \\
\hline \multirow[t]{6}{*}{ Conditional LG-CDM } & Random Effects & $\lambda$ & 0.001 & $0.3 \%$ & 0.001 & -0.002 & $0.4 \%$ & 0.001 \\
\hline & & $h_{k}$ & 0.002 & $0.7 \%$ & 0.003 & -0.003 & $1.0 \%$ & 0.004 \\
\hline & Attribute Difficulty & $b_{k}$ & 0.000 & $18.2 \%$ & 0.022 & 0.000 & $16.9 \%$ & 0.002 \\
\hline & Intervention Effect & $h_{k}$ & 0.002 & $37.4 \%$ & 0.018 & -0.001 & $4.0 \%$ & 0.018 \\
\hline & Item & $f_{j}$ & -0.009 & $1.6 \%$ & 0.022 & -0.002 & $0.7 \%$ & 0.007 \\
\hline & & $d_{j}$ & 0.011 & $1.5 \%$ & 0.030 & 0.003 & $0.5 \%$ & 0.010 \\
\hline
\end{tabular}

Simulation based on 100 data replications.

Unconditional LG-CDM and Conditional LG-CDM and fit with incorrect models one-off, including RDINA model and RDINA model with covariate. The RDINA and RDINA with covariate models do not have a longitudinal component, thereby providing a relative comparison for ignoring the longitudinal component to the model. Model fit indices, $P_{c}$ and \% bias of parameters (random effects, intervention effect, item, and attribute) are presented in the Table 7. All the statistics selected the correct models. For the sample size of 2000, the correct models had higher $P_{c}$, lower AIC/BIC value as well as lower $\%$ bias in both item and attribute parameter estimates. Meanwhile, when fitting with the incorrect models, lower $P_{c}$, higher AIC/BIC and higher \% bias were shown in the outputs. The greatest impact of fitting incorrect models was found in the $\%$ bias of attribute and item parameters. When using Conditional LG-CDM generated data and fit with the RDINA with covariates, \% bias was more than $101 \%$ for the item parameter and attribute difficulty parameter. Similarly, when fitting generated data with RDINA, \% bias were also high for the attribute parameter. It is noticeable when data generated using Conditional LG-CDM were fit using Unconditional LG-CDM, $\%$ bias are lower than the incorrect RDINA and RDINA with covariate model.

TABLE 6 | Simulation classification I: proportion correctly classified $\left(P_{C}\right)$ and Lambda $(\lambda)$.

\begin{tabular}{lccccc}
\hline Models & Classification & $\alpha_{\mathbf{1}}$ & $\alpha_{\mathbf{2}}$ & $\alpha_{\mathbf{3}}$ & $\boldsymbol{\alpha}_{\mathbf{4}}$ \\
\hline Unconditional LG-CDM $(n=1,000)$ & $P_{C}$ & 0.91 & 0.98 & 0.94 & 0.94 \\
& $\lambda$ & 0.80 & 0.95 & 0.87 & 0.84 \\
Conditional LG-CDM $(n=1,000)$ & $P_{C}$ & 0.91 & 0.98 & 0.94 & 0.94 \\
& $\lambda$ & 0.80 & 0.94 & 0.87 & 0.84 \\
Unconditional LG-CDM $(n=2,000)$ & $P_{C}$ & 0.97 & 0.99 & 0.98 & 0.98 \\
& $\lambda$ & 0.93 & 0.99 & 0.97 & 0.96 \\
Conditional LG-CDM $(n=2,000)$ & $P_{C}$ & 0.97 & 0.99 & 0.98 & 0.98 \\
& $\lambda$ & 0.93 & 0.99 & 0.97 & 0.96 \\
\hline
\end{tabular}

Classification statistics based on Clogg (1995).

\section{SIMULATION STUDY II}

\section{Methods}

We conducted an additional simulation study, where three time points were simulated to examine parameter recovery and classification of the proposed Unconditional and Conditional LG-CDMs. As the data of simulation study I were generated using population (true) values derived from the empirical data that was limited to two time points, the condition was expanded to include more time points in simulation study II so that the potentials of the Unconditional and Conditional LG-CDMs can be fully discussed. In simulation study II, to generate data, we referred to the simulation study design conducted by De La Torre and Douglas (2004) to specify the Q-matrix and item parameters, as generating population (true) value: 30 items with five attributes and 1,000 examinees were used across 100 replications. Table 8 shows the transposed Q-matrix that each attribute appears alone, in pair, or in a triple the same number of times as other attributes. Similar to Simulation Study I, 100 data replications were generated and fit using Latent GOLD 5.0 (Vermunt and Magidson, 2013). Parameters were estimated using PM full name estimation and the parameter recovery were evaluated for each condition using three measures: (a) Bias, (b) \% Bias, and (c) mean square error (MSE).

\section{Results}

\section{Parameter Recovery}

Table 9 shows the parameter recovery results of three time points simulation by sample size 1,000 for the two models. Bias, \% bias and MSE were lower for item level parameters (discrimination parameter $d_{j}$ and false rate parameter $f_{j}$ ) in the two models, indicating model estimates are consistent in the item level. The results of parameter recovery for attribute difficulty are slightly high as well as for the intervention effect on attribute difficulty. Furthermore, the bias and \% bias of random effects (random intercept and random slope) are noticeably high in both models, probably because more time points are involved 
TABLE 7 | Cross fitting of simulated data.

\begin{tabular}{|c|c|c|c|}
\hline \multirow[t]{2}{*}{ Data fit } & \multirow[t]{2}{*}{ Statistics } & \multicolumn{2}{|c|}{ Data generating conditions (Sample $n=2,000$ ) } \\
\hline & & Unconditional LG-CDM & Conditional LG-CDM \\
\hline \multirow[t]{5}{*}{ RDINA } & $\mathrm{AlC}$ & 98964.26 & 98945.77 \\
\hline & $\mathrm{BIC}$ & 99234.79 & 99235.29 \\
\hline & $P_{C}$ & 0.87 & 0.87 \\
\hline & Attribute Difficulty (\% Bias) & $91.1 \%$ & $88.4 \%$ \\
\hline & Item (\% Bias) & $28.1 \%$ & $28.0 \%$ \\
\hline \multirow[t]{6}{*}{ RDINA with covariates } & $\mathrm{AIC}$ & - & 98847.14 \\
\hline & $\mathrm{BIC}$ & - & 99161.84 \\
\hline & $P_{C}$ & - & 0.87 \\
\hline & Attribute Difficulty (\% Bias) & - & $101.1 \%$ \\
\hline & Intervention Effect (\% Bias) & - & $64.5 \%$ \\
\hline & Item (\% Bias) & - & $101.2 \%$ \\
\hline \multirow[t]{6}{*}{ Unconditional LG-CDM } & $\mathrm{AlC}$ & 91921.67 & 98728.2 \\
\hline & $\mathrm{BIC}$ & 92157.24 & 98997.04 \\
\hline & $P_{C}$ & 0.98 & 0.97 \\
\hline & Random Effects (\% Bias) & $0.5 \%$ & $13.3 \%$ \\
\hline & Attribute Difficulty (\% Bias) & $3.1 \%$ & $46.1 \%$ \\
\hline & Item (\% Bias) & $0.5 \%$ & $1.1 \%$ \\
\hline \multirow[t]{7}{*}{ Conditional LG-CDM } & $\mathrm{AlC}$ & - & 91971.63 \\
\hline & $\mathrm{BIC}$ & - & 92236.65 \\
\hline & $P_{C}$ & - & 0.98 \\
\hline & Random Effects (\% Bias) & - & $0.7 \%$ \\
\hline & Attribute Difficulty (\% Bias) & - & $16.9 \%$ \\
\hline & Intervention Effect (\% Bias) & - & $4.0 \%$ \\
\hline & Item (\% Bias) & - & $0.6 \%$ \\
\hline
\end{tabular}

Results of the correct model fit were bolded.

in the simulation study that ask for more estimations to reach consistent recovery.

\section{Classification}

Table 10 summarized classification results of three time points simulation. For both LG-CDMs, $P_{c}$ estimates suggested satisfactory proportion of cases are correctly classified for

TABLE 8 | The transposed Q-matrix for the simulation study II.

\begin{tabular}{llllllllllllllll}
\hline & & \multicolumn{1}{c}{ Item } \\
\hline Attribute & $\mathbf{1}$ & $\mathbf{2}$ & $\mathbf{3}$ & $\mathbf{4}$ & $\mathbf{5}$ & $\mathbf{6}$ & $\mathbf{7}$ & $\mathbf{8}$ & $\mathbf{9}$ & $\mathbf{1 0}$ & $\mathbf{1 1}$ & $\mathbf{1 2}$ & $\mathbf{1 3}$ & $\mathbf{1 4}$ & $\mathbf{1 5}$ \\
\hline 1 & 1 & 0 & 0 & 0 & 0 & 1 & 0 & 0 & 0 & 0 & 1 & 1 & 1 & 1 & 0 \\
2 & 0 & 1 & 0 & 0 & 0 & 0 & 1 & 0 & 0 & 0 & 1 & 0 & 0 & 0 & 1 \\
3 & 0 & 0 & 1 & 0 & 0 & 0 & 0 & 1 & 0 & 0 & 0 & 1 & 0 & 0 & 1 \\
4 & 0 & 0 & 0 & 1 & 0 & 0 & 0 & 0 & 1 & 0 & 0 & 0 & 1 & 0 & 0 \\
5 & 0 & 0 & 0 & 0 & 1 & 0 & 0 & 0 & 0 & 1 & 0 & 0 & 0 & 1 & 0 \\
\hline Attribute & $\mathbf{1 6}$ & $\mathbf{1 7}$ & $\mathbf{1 8}$ & $\mathbf{1 9}$ & $\mathbf{2 0}$ & $\mathbf{2 1}$ & $\mathbf{2 2}$ & $\mathbf{2 3}$ & $\mathbf{2 4}$ & $\mathbf{2 5}$ & $\mathbf{2 6}$ & $\mathbf{2 7}$ & $\mathbf{2 8}$ & $\mathbf{2 9}$ & $\mathbf{3 0}$ \\
\hline 1 & 0 & 0 & 0 & 0 & 0 & 1 & 1 & 1 & 1 & 1 & 1 & 0 & 0 & 0 & 0 \\
2 & 1 & 1 & 0 & 0 & 0 & 1 & 1 & 1 & 0 & 0 & 0 & 1 & 1 & 1 & 0 \\
3 & 0 & 0 & 1 & 1 & 0 & 1 & 0 & 0 & 1 & 1 & 0 & 1 & 1 & 0 & 1 \\
4 & 1 & 0 & 1 & 0 & 1 & 0 & 1 & 0 & 1 & 0 & 1 & 1 & 0 & 1 & 1 \\
5 & 0 & 1 & 0 & 1 & 1 & 0 & 0 & 1 & 0 & 1 & 1 & 0 & 1 & 1 & 1
\end{tabular}

all attributes $\left(P_{c}>0.85\right)$. Yet, it should be noted that the classification rates of $\lambda$ were lower on the second and fourth attributes compared to the other attributes, which may due to the over correction for classification error.

TABLE 9 | Simulation I| results: parameter recovery.

\begin{tabular}{|c|c|c|c|c|c|}
\hline \multirow[t]{2}{*}{ Model } & \multirow[t]{2}{*}{ Level } & \multirow[t]{2}{*}{ Parameter } & \multicolumn{3}{|c|}{ Sample $n=1,000$} \\
\hline & & & Bias & $\%$ Bias & MSE \\
\hline \multirow[t]{5}{*}{ Unconditional LG-CDM } & Random Intercept & $\lambda_{0}$ & -0.592 & $118.4 \%$ & 0.354 \\
\hline & Random Slope & $\lambda_{1}$ & 0.312 & $62.4 \%$ & 0.098 \\
\hline & Attribute Difficulty & $b_{k}$ & -0.311 & $39.0 \%$ & 0.123 \\
\hline & Item & $f_{j}$ & 0.073 & $5.1 \%$ & 0.059 \\
\hline & & $d_{j}$ & -0.046 & $2.7 \%$ & 0.088 \\
\hline \multirow[t]{8}{*}{ Conditional LG-CDM } & Random Intercept & $\lambda_{0}$ & -0.548 & $109.6 \%$ & 0.304 \\
\hline & & $h_{0 k}$ & 0.035 & $11.8 \%$ & 0.007 \\
\hline & Random Slope & $\lambda_{1}$ & 0.315 & $63.1 \%$ & 0.101 \\
\hline & & $h_{1 k}$ & -0.222 & $74.1 \%$ & 0.053 \\
\hline & Attribute Difficulty & $b_{k}$ & -0.297 & $37.7 \%$ & 0.122 \\
\hline & Intervention Effect & $h_{k}$ & 0.001 & $20.6 \%$ & 0.173 \\
\hline & Item & $f_{j}$ & 0.065 & $4.7 \%$ & 0.058 \\
\hline & & $d_{j}$ & -0.038 & $2.5 \%$ & 0.082 \\
\hline
\end{tabular}

Simulation based on 100 data replications. 
TABLE 10 | Simulation classification II: proportion correctly classified $\left(P_{C}\right)$ and lambda $(\lambda)$.

\begin{tabular}{lcccccc}
\hline Models & Classification & $\alpha_{\mathbf{1}}$ & $\alpha_{\mathbf{2}}$ & $\alpha_{\mathbf{3}}$ & $\alpha_{\mathbf{4}}$ & $\alpha_{\mathbf{5}}$ \\
\hline Unconditional LG-CDM $(n=1,000)$ & $P_{C}$ & 0.89 & 0.86 & 0.98 & 0.96 & 0.95 \\
& $\lambda$ & 0.72 & 0.47 & 0.79 & 0.36 & 0.76 \\
Conditional LG-CDM $(n=1,000)$ & $P_{C}$ & 0.90 & 0.88 & 0.98 & 0.97 & 0.94 \\
& $\lambda$ & 0.76 & 0.50 & 0.81 & 0.35 & 0.81
\end{tabular}

Classification statistics based on Clogg (1995).

\section{DISCUSSION AND CONCLUSION}

Cognitive diagnostic models have become increasingly important in educational measurement by estimating skill profiles that indicate the examinee's mastery in fine-grained skills based on their performance (Rupp et al., 2010). In most prior studies, CDMs have been applied to single cross-sectional time diagnosis instead of tracking the changes in skills or attributes. However, learning is a process during which students acquire knowledge and improve their skills. As learning progress, students' skills mastery and knowledge could change over time. In addition, the implementation of particular intervention may influence students' learning trajectory, which is important for educators to know in order to evaluate learning and instruction. In this study, we propose two latent growth CDMs, Unconditional LGCDM and Conditional LG-CDM, to assess students' change in skills mastery over time and evaluate the intervention effect on the growth of skill mastery.

Results from the real-world data analysis showed that the latent growth curve model and covariate extension such as intervention effect, could be used to link with a CDM. The statistics of model classification and attribute prevalence agree very much and are excellent for the two LG-CDMs, indicating both models are well specified to provide consistency results. In particular, although the latent class size of Attribute 1 and Attribute 3 are slightly lower than Attribute 2 and Attribute 4, more than half of the students mastered each attribute. Moreover, results showed that the estimates of attribute difficulty of the Conditional LG-CDM was generally lower than the estimates of the Unconditional LG-CDM. The decrease in attribute difficulty indicated that the attributes have been shifted and implies that it become easier for students to master the attributes when involving educational intervention, which was further confirmed by the results of growth curve and intervention effect parameters. Both the Unconditional LG-CDM and Conditional LG-CDM examined the students' performance at baseline and the growth rate. Although the baseline performance is slightly different, the grow rates are similar across the two models. Thus, the LG-CDMs could inform the researchers and educators that the EAI method has little effect on the growth rate of student' ability. However, with the help of Conditional LG$\mathrm{CDM}$ that incorporate the covariates extension into CDM, we can tell that the treatment EAI method does improve students' mastery on the attribute 1 and attribute 4 . In other words, if the students were assigned to the treatment group that receiving the EAI teaching method, they would show progress in math learning, especially in the skills of geometry and ratio/proportional relationships.

The simulation studies showed that the parameters were consistently recovered in general, indicating that incorporating latent growth curve and covariate extension at the attribute level did not affect model estimation. In simulation study I, both attribute and item level estimates were stable with sample size of 1,000 and 2,000 for the Unconditional LG-CDM. For the Conditional LG-CDM, additional attention may be given to the attribute difficulty parameter $\left(b_{k}\right)$ and intervention effect parameter $\left(h_{k}\right)$, percentage bias of $18.2 \%$ and $37.4 \%$ for the sample size of 1,000 . However, with the sample size increase to 2,000 , the $\%$ bias of intervention effect declines rapidly to $4 \%$. The recovery of random parameters was excellent across two models, with bias of random intercepts are less than $2.0 \%$ in the sample size of 1,000 and 2,000.

In Simulation Study II, more time points and items were involved to fully examine the performance of proposed LGCDMs in terms of parameter recovery. The item level estimates were satisfactory with sample size of 1,000 . Although the bias and $\%$ bias of attribute difficulty parameter and intervention effect parameters are slightly high, it is expected that they would decrease obviously when the sample size increase. However, it should be noted that the recovery of random effects for data with three time-point specifications were modest and may depend on study design. Thus, latent growth models may need more specifications or constrains on random intercept and random slope parameters to achieve stable recovery. Besides, the classification indices of the both simulation studies were consistent across different conditions. The results obtained from this study help to advance CDMs to better measuring the change in learning over time.

Researchers and educators have long used pre-post assessment to evaluate the effects of new curriculum and teaching method on students' learning. Meanwhile, it is important to know students' learning trajectory to achieve learning goal. Cognitive diagnostic models have provided a diagnostic framework to measure students' mastery in fine-grained skills and different kinds of longitudinal analysis have been incorporate to the CDM to evaluate changes in skills profile mastery (e.g., Li et al., 2016; Kaya and Leite, 2017). Different from other longitudinal CDMs, the LG-CDMs described in this article incorporate wellestablished latent growth curve model that is more widely used in the social studies. Additionally, covariate extension was integrated to signify the intervention effect. Dayton and Macready (1988) introduced the use of covariate to affect the attribute. Park et al. (2017) included both observed and latent explanatory variables as covariates in the explanatory CDM to inform learning and practice. Thus, this approach is meaningful in the CDM for its diagnostic purpose. In this study, latent growth curve and covariates are both specified at the attribute level. In addition, latent growth curve could be also specified at the item level, contributing to the multilevel studies on CDMs. Meanwhile, more attributes and covariates could be incorporated in specifying the items and explaining the relationship among them. In the empirical study, it is likely to have items that are of high slip and guessing estimates in the test (Lee et al., 2011), 
therefore, it is important for the researchers to carefully develop and validate the Q-matrix used for CDM analyses.

For the future studies, different types of variance-covariance structures could be specified in the model. For example, all the parameters could be freely estimated (unstructured) in the covariance structure to explore their relationships in model estimation. Meanwhile, future studies could conduct a comprehensive investigation of the measurement invariance, building on the foundational simulation studies conducted in this paper. For example, additional parameters could be included to examine their effects on model identification and their impact on the measurement invariance. Furthermore, the item level fit statistics could be developed in the future studies for the itemlevel effects in the LG-CDMs, which provides suitable item level fit information for the studies that involve multiple time points. In the current field of education, individualized learning has been emphasized, which allows students to construct learning progress at own pace. This study provides a flexible framework to diagnose skill mastery as well as advancing the longitudinal CDMs to better measuring the change in learning over time.

\section{DATA AVAILABILITY STATEMENT}

The data analyzed in this study is subject to the following licenses/restrictions: The use of datasets requires permission.

\section{REFERENCES}

Andersen, E. B. (1985). Estimating latent correlations between repeated testings. Psychometrika 50, 3-16. doi: 10.1007/bf02294143

Bottge, B. A., Heinrichs, M., Chan, S.-Y., Mehta, Z. D., and Watson, E. (2003). Effects of video-based and applied problems on the procedural math skills of average-and low-achieving adolescents. J. Special Educ. Technol. 18, 5-22. doi: 10.1177/016264340301800201

Bottge, B. A., Ma, X., Gassaway, L., Toland, M., Butler, M., and Cho, S. J. (2014). Effects of blended instructional models on math performance. Except. Child. 80, 237-255. doi: 10.1177/0014402914527240

Bottge, B. A., Toland, M., Gassaway, L., Butler, M., Choo, S., Griffen, A. K., et al. (2015). Impact of enhanced anchored instruction in inclusive math classrooms. Except. Child. 81, 158-175. doi: 10.1177/0014402914551742

Bradshaw, L., Izsák, A., Templin, J., and Jacobson, E. (2014). Diagnosing teachers' understandings of rational numbers: building a multidimensional test within the diagnostic classification framework. Educ. Meas. Issues Pract. 33, 2-14. doi: 10.1111 /emip. 12020

Bradshaw, L., and Levy, R. (2019). Interpreting probabilistic classifications from diagnostic psychometric models. Educ. Meas. Issues Pract. 38, 79-88. doi: 10.1111/emip. 12247

Chen, Y., Culpepper, S. A., Wang, S., and Douglas, J. (2018). A hidden Markov model for learning trajectories in cognitive diagnosis with application to spatial rotation skills. Appl. Psychol. Meas. 42, 5-23. doi: 10.1177/01466216177 21250

Clogg, C. C. (1995). "Latent class models," in Handbook of Statistical Modeling for the Social and Behavioral Sciences, eds G. Arminger, C. C. Clogg, and M. E. Sobel (Boston, MA: Springer), 311-359.

Collins, L. M., and Wugalter, S. E. (1992). Latent class models for stage-sequential dynamic latent variables. Multivar. Behav. Res. 27, 131-157. doi: 10.1207/ s15327906mbr2701_8

Culpepper, S. A. (2019). An exploratory diagnostic model for ordinal responses with binary attributes: identifiability and estimation. Psychometrika 84, 921940. doi: 10.1007/s11336-019-09683-4
Requests to access these datasets should be directed to University of Kentucky.

\section{AUTHOR CONTRIBUTIONS}

QL contributed to the conceptualization of the manuscript and conducted formal analysis as well as wrote the original draft. KX contributed to the review and edition of the manuscript. YP contributed to the supervision, conceptualization, and review of the manuscript. All authors contributed to the article and approved the submitted version.

\section{FUNDING}

This research was supported by the Institute of Education Sciences (IES) grant R324A150035.

\section{ACKNOWLEDGMENTS}

The opinions expressed are those of the authors and do not necessarily reflect the views of IES.

Culpepper, S. A., and Chen, Y. (2019). Development and application of an exploratory reduced reparameterized unified model. J. Educ. Behav. Stat. 44, 3-24. doi: 10.3102/1076998618791306

Dayton, C. M., and Macready, G. B. (1988). "A latent class covariate model with applications to criterion-referenced testing," in Latent Trait and Latent Class Models, eds R. Langeheine, and J. Rost (Boston, MA: Springer), 129-143. doi: 10.1007/978-1-4757-5644-9_7

de la Torre, J. (2011). The generalized DINA model framework. Psychometrika 76, 179-199. doi: 10.1007/s11336-011-9207-7

De La Torre, J., and Douglas, J. A. (2004). Higher-order latent trait models for cognitive diagnosis. Psychometrika 69, 333-353. doi: $10.1007 \backslash \% 2$ FBF02295640

DeCarlo, L. T. (2011). On the analysis of fraction subtraction data: the DINA model, classification, latent class sizes, and the Q-matrix. Appl. Psychol. Meas. 35, 8-26. doi: 10.1177/0146621610377081

DiBello, L. V., Stout, W. F., and Roussos, L. A. (1995). "Unified cognitive/psychometric diagnostic assessment likelihood-based classification techniques," in Cognitively Diagnostic Assessment, eds P. Nichols, S. Chipman, and R. Brennen (Hillsdale, NJ: Earlbaum), 361-389.

Duncan, T. E., Duncan, S. C., and Strycker, L. A. (2013). An Introduction to Latent Variable Growth Curve Modeling: Concepts, Issues, and Application. Abingdon: Routledge.

Embretson, S. E. (1991). A multidimensional latent trait model for measuring learning and change. Psychometrika 56, 495-515. doi: 10.1007/bf02294487

Fischer, G. H. (1989). An IRT-based model for dichotomous longitudinal data. Psychometrika 54, 599-624. doi: 10.1007/bf0229 6399

Henson, R. A., Templin, J. L., and Willse, J. T. (2009). Defining a family of cognitive diagnosis models using log-linear models with latent variables. Psychometrika 74:191. doi: 10.1007/s11336-008-9089-5

Huang, G. H., and Bandeen-Roche, K. (2004). Building an identifiable latent class model with covariate effects on underlying and measured variables. Psychometrika 69, 5-32. doi: 10.1007/BF02295837

Huang, H. Y. (2017). Multilevel cognitive diagnosis models for assessing changes in latent attributes. J. Educ. Meas. 54, 440-480. doi: 10.1111/jedm.12156 
Junker, B. W., and Sijtsma, K. (2001). Cognitive assessment models with few assumptions, and connections with nonparametric item response theory. Appl. Psychol. Meas. 25, 258-272. doi: 10.1177/01466210122032064

Kaya, Y., and Leite, W. L. (2017). Assessing change in latent skills across time with longitudinal cognitive diagnosis modeling: an evaluation of model performance. Educ. Psychol. Meas. 77, 369-388. doi: 10.1177/ 0013164416659314

Kruskal, W. H., and Goodman, L. (1954). Measures of association for cross classifications. J. Am. Stat. Assoc. 49, 732-764. doi: 10.1007/978-1-4612-9995-0

Lee, S. Y. (2017). Growth Curve Cognitive Diagnosis Models for Longitudinal Assessment. Berkeley, CA: UC Berkeley.

Lee, Y.-S., Park, Y. S., and Taylan, D. (2011). A cognitive diagnostic modeling of attribute mastery in Massachusetts, Minnesota, and the US national sample using the TIMSS 2007. Int. J. Testing 11, 144-177. doi: 10.1080/15305058.2010. 534571

Li, F., Cohen, A., Bottge, B., and Templin, J. (2016). A latent transition analysis model for assessing change in cognitive skills. Educ. Psychol. Meas. 76, 181-204. doi: $10.1177 / 0013164415588946$

Linn, R. L., and Slinde, J. A. (1977). The determination of the significance of change between pre-and posttesting periods. Rev. Educ. Res. 47, 121-150. doi: 10.3102/00346543047001121

Madison, M., and Bradshaw, L. (2018a). Assessing growth in a diagnostic classification model framework. Psychometrika 83, 963-990. doi: 10.1007/ s11336-018-9638-5

Madison, M., and Bradshaw, L. (2018b). Evaluating intervention effects in a diagnostic classification model framework. J. Educ. Meas. 55, 32-51. doi: 10. $1111 /$ jedm. 12162

Meredith, W., and Tisak, J. (1984). "Statistical considerations in Tuckerizing curves with emphasis on growth curves and cohort sequential analysis," in Annual Meeting of the Psychometric Society.

Meredith, W., and Tisak, J. (1990). Latent curve analysis. Psychometrika 55, 107-122. doi: 10.1007/BF02294746

Park, Y. S., and Lee, Y.-S. (2014). An extension of the DINA model using covariates: examining factors affecting response probability and latent classification. Appl. Psychol. Meas. 38, 376-390. doi: 10.1177/0146621614523830

Park, Y. S., and Lee, Y.-S. (2019). "Explanatory cognitive diagnostic models," in Handbook of Diagnostic Classification Models, eds M. von Davier, and Y. S. Lee, (Berlin: Springer), 207-222. doi: 10.1007/978-3-030-05584-4_10

Park, Y. S., Xing, K., and Lee, Y.-S. (2017). Explanatory cognitive diagnostic models: incorporating latent and observed predictors. Appl. Psychol. Meas. 42, 376-392. doi: $10.1177 / 0146621617738012$
Rao, C. R. (1958). Some statistical methods for comparison of growth curves. Biometrics 14, 1-17. doi: 10.2307/2527726

Rupp, A. A., Templin, J., and Henson, R. A. (2010). Diagnostic Measurement: Theory, Methods, and Applications. New York, NY: Guilford Press.

Tatsuoka, K. K. (1985). A probabilistic model for diagnosing misconceptions by the pattern classification approach. J. Educ. Stat. 10, 55-73. doi: 10.3102/ 10769986010001055

Templin, J. L., and Henson, R. A. (2006). Measurement of psychological disorders using cognitive diagnosis models. Psychol. Methods 11:287. doi: 10.1037/1082989x.11.3.287

Tucker, L. R. (1958). Determination of parameters of a functional relation by factor analysis. Psychometrika 23, 19-23. doi: 10.1007/BF02288975

Vermunt, J. K., and Magidson, J. (2013). Technical Guide for Latent GOLD 5.0: Basic, Advanced, and Syntax. Belmont, MA: Statistical Innovations Inc.

von Davier, M. (2005). A general diagnostic model applied to language testing data. ETS Res. Rep. Series 2005, i-35.

von Davier, M. (2008). A general diagnostic model applied to language testing data. Br. J. Math. Stat. Psychol. 61, 287-307. doi: 10.1348/000711007x19 3957

von Davier, M. (2014). The log-linear cognitive diagnostic model (LCDM) as a special case of the general diagnostic model (GDM). ETS Res. Rep. Series 2014, 1-13. doi: 10.1002/ets2.12043

Wang, S., Yang, Y., Culpepper, S. A., and Douglas, J. A. (2018). Tracking skill acquisition with cognitive diagnosis models: a higher-order, hidden markov model with covariates. J. Educ. Behav. Stat. 43, 57-87. doi: 10.3102/ 1076998617719727

Zhan, P., Jiao, H., Liao, D., and Li, F. (2019). A longitudinal higher-order diagnostic classification model. J. Educ. Behav. Stat. 44, 251-281. doi: 10.3102/ 1076998619827593

Conflict of Interest: The authors declare that the research was conducted in the absence of any commercial or financial relationships that could be construed as a potential conflict of interest.

Copyright (c) 2020 Lin, Xing and Park. This is an open-access article distributed under the terms of the Creative Commons Attribution License (CC BY). The use, distribution or reproduction in other forums is permitted, provided the original author(s) and the copyright owner(s) are credited and that the original publication in this journal is cited, in accordance with accepted academic practice. No use, distribution or reproduction is permitted which does not comply with these terms. 


\section{APPENDIX}

TABLE A1 | The Q-matrix for the real-world data analysis.

\begin{tabular}{|c|c|c|c|c|}
\hline \multirow[b]{2}{*}{ Item } & \multicolumn{4}{|c|}{ Attribute } \\
\hline & $\alpha_{1}$ & $\alpha_{2}$ & $\alpha_{3}$ & $\alpha_{4}$ \\
\hline 1 & 1 & 0 & 0 & 0 \\
\hline 2 & 0 & 1 & 0 & 0 \\
\hline 3 & 0 & 1 & 0 & 0 \\
\hline 4 & 0 & 0 & 1 & 0 \\
\hline 5 & 0 & 0 & 1 & 0 \\
\hline 6 & 0 & 0 & 1 & 0 \\
\hline 7 & 0 & 0 & 1 & 0 \\
\hline 8 & 0 & 0 & 1 & 0 \\
\hline 9 & 0 & 1 & 0 & 0 \\
\hline 10 & 0 & 1 & 0 & 0 \\
\hline 11 & 0 & 1 & 0 & 0 \\
\hline 12 & 0 & 1 & 0 & 0 \\
\hline 13 & 1 & 0 & 0 & 0 \\
\hline 14 & 1 & 0 & 0 & 0 \\
\hline 15 & 0 & 0 & 0 & 1 \\
\hline 16 & 0 & 0 & 0 & 1 \\
\hline 17 & 1 & 0 & 0 & 0 \\
\hline 18 & 0 & 0 & 0 & 1 \\
\hline 19 & 0 & 0 & 0 & 1 \\
\hline 20 & 0 & 0 & 0 & 1 \\
\hline 21 & 0 & 0 & 0 & 1 \\
\hline
\end{tabular}

